\title{
EMBEDDING FINITE SEMIGROUP AMALGAMS
}

\author{
JAN OKNIŃSKI and MOHAN S. PUTCHA
}

(Received 12 February 1990)

Communicated by P. G. Trotter

\begin{abstract}
Let $S, T_{1}, \ldots, T_{k}$ be finite semigroups and $\varphi_{i}: S \hookrightarrow T_{i}$ be embeddings. When $\mathbb{C}[S]$ is semisimple, we find necessary and sufficient conditions for the semigroup amalgam $\left(T_{1}, \ldots, T_{k} ; S\right)$ to be embeddable in a finite semigroup. As a consequence we show that if $S$ is a finite semigroup with $\mathbb{C}[S]$ semisimple, then $S$ is an amalgamation base for the class of finite semigroups if and only if the principal ideals of $S$ are linearly ordered. Our proof uses both the theory of representations by transformations and the theory of matrix representations as developed by Clifford, Munn and Ponizovskii
\end{abstract}

1991 Mathematics subject classification (Amer. Math. Soc.) 20 M 10, 20 M 30, 20M 25.

\section{Introduction}

While the theory of matrix representations has played a critical role in finite group theory, the same is not so far true in finite semigroup theory. In this paper we give an application of linear representation theory to the study of finite semigroup amalgams.

A basic open problem in finite semigroup theory is to determine when a finite semigroup $S$ is an amalgamation base for the class of finite semigroups. It was shown by Hall and one of the authors [6] that a necessary condition is that the $\mathscr{J}$-classes of $S$ are linearly ordered. Then Hall [5] showed that any finite inverse semigroup with linearly ordered $\mathscr{T}$-classes is an amalgamtion base for the class of finite inverse semigroups. Moreover, he conjectured (private communication) that such a semigroup is also an amalgamation base for the class of finite semigroups. For combinatorial inverse semigroups, this

(C) 1991 Australian Mathematical Society 0263-6115/91\$A2.00+0.00 
was established by Hall and the second named author in 1987 (unpublished). At the same time the author was informed by Hall that he could also prove the result for finite groups. In this paper we establish a much more general result, namely that if $S$ is any finite semigroup with linearly ordered $\mathscr{T}$-classes and the semigroup algebra $\mathbb{C}[S]$ semisimple, then $S$ is an amalgamation base for the class of finite semigroups. Combining this with an earlier result of the authors [8], it follows that the multiplicative semigroup $\mathscr{K}_{n}\left(\mathbb{F}_{q}\right)$ is an amalgamation base for the class of finite semigroups.

A related open problem is to find necessary and sufficient conditions for embedding a finite semigroup amalgam into a finite semigroup. Again we solve the problem when $\mathbb{C}[S]$ is semisimple, where $S$ is the core semigroup. The conditions are in terms of the associated maps between the partially ordered sets of regular $\mathscr{T}$-classes.

\section{Main results}

Let $S$ be a finite semigroup. If $Y \subseteq S$, then we let

$$
E(Y)=\left\{e \in X \mid e^{2}=e\right\} .
$$

We let $\mathscr{U}(S)$ denote the partially ordered set of regular $\mathscr{T}$-classes of $S$. If $P, Q$ are partially ordered sets, then a map $\theta: P \rightarrow Q$ is strict order preserving if for all $a, b \in P, a<b$ implies $\theta(a)<\theta(b)$. If $S, T$ are finite semigroups and $\varphi: S \hookrightarrow T$ is an embedding, then the induced map $\widehat{\varphi}: \mathscr{U}(S) \rightarrow \mathscr{U}(T)$ is strict order preserving.

If a finite semigroup $S$ acts (on the left) on a finite set $X$, then as usual we say that $X$ is an $S$-system. Let $X$ be a $G$-system where $G$ is a finite group with identity element $e$. Then $Y=e X$ is a disjoint union of $G$-orbits $Y_{1}, \ldots, Y_{t}$. Let

$$
X_{i}=\left\{x \in X \mid e x \in Y_{i}\right\}, i=1, \ldots, t .
$$

Then $X$ is a disjoint union of $X_{1}, \ldots, X_{t}$. We call $X_{i}$ a pseudo-orbit. Let

$$
a \in Y_{i}, H=\{h \in G \mid h a=a\} .
$$

Then $Y_{i} \cong G / H=\{g H \mid g \in G\}$ as $G$-systems. We will say that $X_{i}$ and $Y_{i}$ are of type $H$. Then the type is uniquely determined up to conjugacy. If $H=G$, then we say that $X_{i}$ is a trivial pseudo-orbit.

Lemma 1. Let $S$ be a finite semigroup, $e \in E(S), G$ the $\mathscr{H}$-class of e. Suppose that $G$ is also a subgroup of a finite group $\widehat{G}$. Then there is 
a finite semigroup $T$ and embeddings $\varphi_{1}: S \hookrightarrow T, \varphi_{2}: \widehat{G} \hookrightarrow T$ such that $\left.\varphi_{1}\right|_{G}=\left.\varphi_{2}\right|_{G}$.

Proof. Let $X$ be a finite set on which $S$ acts faithfully. For a subgroup $H$ of $G$, let $Y_{H}=\widehat{G} / H=\{g H \mid g \in \widehat{G}\}$. Then as a $G$-system, $Y_{H}$ has at least one orbit of type $H$. Let $Y$ denote the disjoint union of $|X|$ copies of $Y_{H}$ as $H$ ranges through all the subgroups of $G$. Then $\widehat{G}$ acts faithfully on $Y_{e}$ and hence on $Y$. Also a $G$-orbit of each type occurs at least $|X|$ times. Let $I=\{a \in S \mid e \notin S a S\}$ and let $V$ be the Rees factor semigroup $S / I$. Then $S$ acts on $W=V e$. For a subgroup $H$ of $G, H \neq G, S$ acts on $W_{H}=\{a H \mid a \in W\}$. As a $G$-system $W_{H}$ has at most two pseudo-orbits: one of type $H$ and possibly a trivial one. Thus by adding the needed copies of $W_{H}$ 's to $X$, we can assume that for each subgroup $H$ of $G, H \neq G$, the number of pseudo-orbits of type $H$ of the $G$-system $X$ is equal to the number of orbits of type $H$ of the $G$-system $Y$. Since we can obviously add the needed number of trivial orbits to $X$ or $Y$, we can assume that the number of trivial pseudo-orbits of $X$ is also equal to the number of trivial orbits of $Y$. Then the $G$-systems $e X$ and $Y$ are isomorphic. So we can assume that $Y=e X$. Then $\widehat{G}$ acts faithfully on $X$ as: $g x=g(e x)$, $x \in X, g \in \widehat{G}$. We can then take $T$ to be the full transformation semigroup on $X$.

Corollary 2. Let $S$ be a finite semigroup, $e \in E(S), G$ the $\mathscr{H}$-class of e. Let $H_{1}, H_{2}$ be subgroups of $G, \sigma: H_{1} \rightarrow H_{2}$ an isomorphism. Then there is a finite semigroup $T$ containing $S$ and an element $x$ in the $\mathscr{H}$-class of $e$ in $T$ such that $x^{-1} h x=\sigma(h)$ for all $h \in H_{1}$.

Proof. By [7, Section 18], there is a finite group $\widehat{G}$ containing $G$ such that $\sigma$ is given by conjugation in $\widehat{G}$. The result now follows from Lemma 1.

Remark 3. Let $F$ be a finite field. Then $T$ embeds into some $\mathscr{M}_{n}(F)$. So there exists $y \in \operatorname{GL}(n, F)$ such that $y^{-1} h y=\sigma(h)$ for all $h \in H_{1}$.

LEMMA 4. Let $T$ be a finite semigroup, $\alpha: \mathscr{U}(T) \rightarrow \mathbb{Z}$ a strict orderpreserving map,

$$
\alpha(\mathscr{U}(T))=\left\{r_{1}<r_{2}<\cdots<r_{k}\right\}
$$

such that if $t=\left|T^{1}\right|$, then $r_{1}>t$ and $r_{i}>r_{i-1} \cdot t \cdot 2^{t}, i=2, \ldots, k$. Then for any field $F$, there is an embedding of $T$ into some $\mathscr{M}_{n}(F)$ such that each $J \in \mathscr{U}(T)$ consists of matrices of rank $\alpha(J)$. 
Proof. Let $\mathscr{U}(T)=\left\{J_{1}, \ldots, J_{m}\right\}$ with $\alpha\left(J_{1}\right) \leq \cdots \leq \alpha\left(J_{m}\right)$. The regular representation of $T$ yields an embedding $\psi: T \hookrightarrow \mathscr{M}_{t}(F)$ where $t=\left|T^{1}\right|$. Let $a_{1}, \ldots, a_{m}$ be the ranks of the matrices in $\psi\left(J_{1}\right), \ldots, \psi\left(J_{m}\right)$, respectively. We use the fact that the $j$ th exterior power $\Lambda^{j}, j=1, \ldots, t$ can

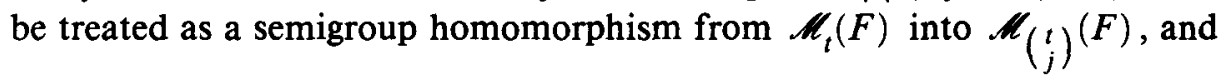
if $A \in \mathscr{H}_{t}(F)$ is a matrix of rank $r$ then $\Lambda^{j}(A)$ has rank $\left(\begin{array}{l}r \\ j\end{array}\right)$ if $r \geq j$ and 0 otherwise. For $i=1, \ldots, m$ let

$$
I_{i}=\left\{x \in T \mid J_{i} \nsubseteq T^{1} x T^{1}\right\}
$$

and $T_{i}$ the Rees factor semigroup $T / I_{i}$. Let $\pi_{i}: T \rightarrow T / I_{i}$ denote the natural homomorphism. Let $t_{i}=\left|T_{i}^{1}\right|$. Then the regular representation of $T_{i}$ induces an embedding $\psi_{i}: T_{i} \hookrightarrow \mathscr{M}_{t_{i}}(F)$. Let $a_{k i}$ denote the rank of the

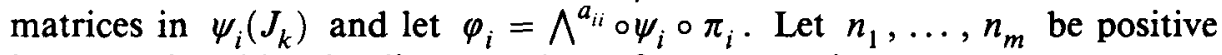
integers. Consider the direct product of representations

$$
\varphi=\psi \oplus \varphi_{1}^{n_{1}} \oplus \cdots \oplus \varphi_{n}^{n_{m}}, \varphi_{i}^{n}
$$

denoting the direct product of $n$ copies of $\varphi_{i}$. This defines an embedding $T \hookrightarrow \mathscr{M}_{n}(F)$ where

$$
n=t+n_{1}\left(\begin{array}{c}
t_{1} \\
a_{11}
\end{array}\right)+\cdots+n_{m}\left(\begin{array}{c}
t_{m} \\
a_{m m}
\end{array}\right) .
$$

Then $\varphi\left(J_{i}\right)$ consists of matrices of rank $a_{i}+\sum n_{j}\left(a_{i j}\right)+n_{i}$ where the sum is taken over all $j$ such that $J_{i}>J_{j}$. Thus, we have to solve the system of equations

$$
a_{i}+\sum n_{j}\left(\begin{array}{c}
a_{i j} \\
a_{j j}
\end{array}\right)+n_{i}=s_{i}=\alpha\left(J_{i}\right), \quad i=1, \ldots, m
$$

for $n_{1}, \ldots, n_{m}$ in the set of positive integers. Since by the hypothesis on $\alpha$, this is a triangular system with diagonal coefficients equal to 1 , this can be inductively solved provided that

$$
s_{1}>a_{1} \text { and } s_{i}>a_{i}+\sum n_{j}\left(\begin{array}{c}
a_{i j} \\
a_{j j}
\end{array}\right) \text { for } i=2, \ldots, m \text {. }
$$

Let $s_{1}, \ldots, s_{l}=r_{1}<s_{l+1}$. Then $J_{1}, \ldots, J_{l}$ are not comparable, so the first $l$ equations look like

$$
r_{1}=a_{i}+n_{i} i=1, \ldots, l .
$$

Since $r_{1}>t$, we can choose $n_{i}=r_{1}-a_{i}$. Assume that $s_{q}<s_{q+1}$ and $n_{1}, \ldots, n_{q}$ have been chosen so that the first $q$ equations are satisfied. Let $s_{q+1}=\cdots=s_{p}$ and $s_{p}<s_{p+1}$ if $p \neq m$. Since $J_{q+1}, \ldots, J_{p}$ are not 
comparable, we see that for $i=q+1, \ldots, p, n_{i}$ does not appear in the $j$ th equation for $q+1 \leq j \leq p, j \neq i$. Since $n_{j} \leq s_{j}$ for $j \leq q$, we have

$$
\begin{aligned}
a_{i}+\sum n_{j}\left(\begin{array}{c}
a_{i j} \\
a_{j j}
\end{array}\right) & \leq a_{i}+\sum s_{j} \cdot 2^{a_{i j}} \leq t+\sum s_{q} \cdot 2^{t} \\
& \leq s_{q} \cdot 2^{t} \cdot m \leq s_{q} \cdot 2^{t} \cdot t<s_{q+1}=s_{i}
\end{aligned}
$$

for $i=q+1, \ldots, p$. The result follows.

Finally, we will need the following consequence of matrix representation theory of finite semigroups.

Proposition 5. Let $S$ be a finite semigroup such that the semigroup algebra $F[S]$ is semisimple for a finite field $F$. Assume that $V, W$ are finite dimensional left $F[S]$-modules of the same dimension such that, for every $e=e^{2} \in S$ and the $\mathscr{H}$-class $H$ of $e$ in $S, e V$ and $e W$ are isomorphic as $F[H]$-modules. Then $V, W$ are isomorphic $F[S]$-modules.

Proof. Let the $\mathscr{T}$-classes of $S$ be listed as $J_{1}, \ldots, J_{t}$ so that $J_{i} \ngtr J_{j}$ for $i<j$. Let $e_{i}=e_{i}^{2} \in J_{i}$. Since $F[S]$ is semisimple, $V, W$ are direct sums of irreducible submodules. It is known that every non-null irreducible $F[S]$-module $Z$ comes from a $\mathscr{T}$-class $J$ of $S$, cf., [2, Theorem 5.33]. Specifically, $Z$ has the induced $F\left[J^{0}\right]$-module structure and $a Z \neq 0$ only if the $\mathscr{T}$-class $J_{a}$ of $a \in S$ satisfies $J_{a} \geq J$. Let

$$
V=X_{1} \oplus \sigma \oplus X_{t} \oplus X \text { and } W=Y_{1} \oplus \cdots \oplus Y_{t} \oplus Y
$$

where

$$
X_{k}=V_{k 1} \oplus \cdots \oplus V_{k i_{k}} \quad \text { and } \quad Y_{k}=W_{k 1} \oplus \cdots \oplus W_{k j_{k}}
$$

are direct sums of non-null irreducible submodules determined by $J_{k}$, and $X, Y$ are trivial submodules. By induction on $k$ we show that $X_{k} \simeq Y_{k}$. Assume that $X_{l} \simeq Y_{l}$ for $l<k$. Then

$$
e_{k} X_{1} \oplus \cdots \oplus e_{k} X_{k-1} \simeq e_{k} Y_{1} \oplus \cdots \oplus e_{k} Y_{k-1}
$$

as $F\left[e_{k} S e_{k}\right]$-modules, and so as $F[H]$-modules for the $\mathscr{H}$-class $H$ of $e_{k}$ in $S$. Since

$$
e_{k} V=e_{k} X_{1} \oplus \cdots \oplus e_{k} X_{k} \quad \text { and } \quad e_{k} W=e_{k} Y_{1} \oplus \cdots \oplus e_{k} Y_{k}
$$

are isomorphic $F[H]$-modules by the hypothesis, it follows that $e_{k} X_{k} \simeq e_{k} Y_{k}$ as $F[H]$-modules. Then $X_{k} \simeq Y_{k}$ as $F[S]$-modules, cf., [2, Section 5.4 and Theorem 5.33]. Since $\operatorname{dim} V=\operatorname{dim} W$, we also have $X \simeq Y$, so that $V \simeq W$. 
Definition. Let $Q, P_{1}, \ldots, P_{k}$ be finite partially ordered sets, $\alpha_{i}: Q \rightarrow$ $P_{i}$ be strict order preserving maps. Then $\alpha_{1}, \ldots, \alpha_{k}$ are compatible if there is a finite partially ordered set $P$, and strict order preserving maps $\beta_{i}: P_{i} \rightarrow P$ such that $\beta_{i} \circ \alpha_{i}=\beta_{j} \circ \alpha_{j}$ for all $i, j$.

REMARK 6. (i) If $\alpha_{1}, \ldots, \alpha_{k}$ are compatible, then $P$ can be chosen to be a finite linearly ordered set since any finite partially ordered set admits a strict order preserving map into a finite linearly ordered set.

(ii) If $Q$ is a linearly ordered set, then $\alpha_{1}, \ldots, \alpha_{k}$ are always compatible.

(iii) If $Q$ is not linearly ordered, then there exist $P_{1}, P_{2}, \alpha_{1}, \alpha_{2}$ which are not compatible.

Let $S, T_{1}, \ldots, T_{k}$ be semigroups with $\varphi_{i}: S \hookrightarrow T_{i}$ embeddings. Then $\left(T_{1}, \ldots, T_{k} ; S\right)$ is called an amalgam and $S$ its core. The amalgam is embeddable in a semigroup $T$ if there exist embeddings $\psi_{i}: T_{i} \hookrightarrow T$ such that $\psi_{i} \circ \varphi_{i}=\psi_{j} \circ \varphi_{j}$ for all $i, j$. We now prove our main theorem.

Theorem 7. Let $S, T_{1}, \ldots, T_{k}$ be finite semigroups with $\varphi_{i}: S \hookrightarrow T_{i}$ embeddings with induced maps $\hat{\varphi}_{i}: \mathscr{U}(S) \rightarrow \mathscr{U}\left(T_{i}\right)$. If $\mathbb{C}[S]$ is semisimple, then the amalgam $\left(T_{1}, \ldots, T_{k} ; S\right)$ is embeddable in a finite semigroup if and only if $\widehat{\varphi}_{1}, \ldots, \widehat{\varphi}_{k}$ are compatible.

Proof. The necessity being obvious, we prove sufficiency. So assume that $\widehat{\varphi}_{1}, \ldots, \widehat{\varphi}_{k}$ are compatible. By [8, Proposition 2.11] there exists a finite field $F$ such that $F[S]$ is semisimple. By Lemma 4 and Remark 6(i), we can assume that $T_{i}=\mathscr{M}_{n}(F)$, for all $i$ and that for all $e \in E(S)$ and all $i, j, \varphi_{i}(e)$ and $\varphi_{j}(e)$ are of the same rank. Let $e \in E(S), H$ the $\mathscr{H}$-class of $e$ in $S$. Fix $i, j$. There is $x \in \mathrm{GL}(n, F)$ such that $x^{-1} \varphi_{j}(e) x=$ $\varphi_{i}(e)$. Then $\sigma: \varphi_{i}(H) \rightarrow x^{-1} \varphi_{j}(H) x$ given by $\sigma\left(\varphi_{i}(g)\right)=x^{-1} \varphi_{j}(g) x$ is an isomorphism. So by Corollary 2 and Remark 3 we can replace $\mathscr{M}_{n}(F)$ by some $\mathscr{M}_{p}(F)$ so that $\sigma$ is given by conjugacy by an element of $\operatorname{GL}(p, F)$. Repeating this argument we can assume without loss of generality that for all $e \in E(S)$ and all $i, j$, there exists $x \in \mathrm{GL}(n, F)$ such that

$$
x^{-1} \varphi_{i}(a) x=\varphi_{j}(a) \text { for all } a \in H_{e}
$$

where $H_{e}$ is the $\mathscr{H}$-class of $e$. Hence by Proposition 5 , the linear representations $\varphi_{1}, \ldots, \varphi_{k}$ are all equivalent. This completes the proof of the theorem.

Remark 8. Let $S$ be a finite semigroup. Munn and Ponizovskii have characterized the semisimplicity of $\mathbb{C}[S]$ in terms of the invertibility of the sandwich matrices over appropriate group algebras, cf., [2, Chapter 5]. In 
particular $\mathbb{C}[S]$ is semisimple for finite inverse semigroups $S$. Recently the authors [8] have shown by a difficult argument that the complex semigroup algebras of finite monoids of Lie type are also semisimple. These are the finite analogues of linear algebraic monoids.

Let $S$ be a finite semigroup. Then $S$ is an amalgamation base for the class of finite semigroups if any finite semigroup amalgam $\left(T_{1}, \ldots, T_{k} ; S\right)$ can be embedded in a finite semigroup. By Remark 6(ii), Theorem 7 and [6] we have:

THEOREM 9. Let $S$ be a finite semigroup with $\mathbb{C}[S]$ semisimple. Then $S$ is an amalgamation base for the class of finite semigroups if and only if the $\mathscr{T}$-classes of $S$ are linearly ordered.

CoROLlaRY 10. A finite inverse semigroup $S$ is an amalgamation base for the class of finite semigroups if and only if the $\mathscr{T}$-classes of $S$ are linearly ordered.

The authors $[8$, Corollary 2.10$]$ have shown that $\mathbb{C}\left[\mathscr{K}_{n}(F)\right]$ is semisimple for any finite field $F$. This result was claimed much earlier by Faddeev [3]. However, his proof is incomplete with no further details ever published or otherwise available. Since $\mathscr{K}_{n}(F)$ has linearly ordered $\mathscr{T}$-classes, we have:

COROLlaRY 11. For any finite field $F$, the multiplicative semigroup $\mathscr{M}_{n}(F)$ is an amalgamation base for the class of finite semigroups.

The problem of determining all (regular) amalgamation bases for the class of finite semigroups remains open. In light of the results of this paper, we feel that the final answer will have something to do with linear representation theory. However by Hall [4], the two element right zero semigroup is an amalgamation base, while the three element right zero semigroup is not. So the exact conditions are not at this point clear to the authors.

\section{References}

[1] N. Bourbaki, Algèbre, Chapitre III (Hermann, Paris, 1948).

[2] A. H. Clifford and G. B. Preston, Algebraic theory of semigroups, (Amer. Math. Soc., Providence, R.I., 1961).

[3] D. K. Faddeev, 'On representations of a full matrix semigroup over a finite field,' Dokl. Akad. Nauk SSSR 230 (1976), 1290-1293.

[4] T. E. Hall, 'Representation extension and amalgamation for semigroups,' Quart. J. Math. Oxford Ser. (2) 29 (1978), 309-334. 
[5] T. E. Hall, 'Finite inverse semigroups and amalgamations,' Semigroups and their applications, pp. 51-56 (Reidel, 1987).

[6] T. E. Hall and M. S. Putcha, 'The potential $J$-relation and amalgamation bases for finite semigroups,' Proc. Amer. Math. Soc. 95 (1985), 361-364.

[7] B. H. Buemann, 'An essay on free products of groups with amalgamations,' Philos. Trans. Roy. Soc. London Ser. A 246 (1954), 503-554.

[8] J. Okniński and M. S. Putcha, 'Complex representations of matrix semigroups,' Trans. Amer. Math. Soc., 323 (1991), 563-581.

North Carolina State University

Raleigh, North Carolina 27695-8205

U.S.A. 